\title{
Design and Evaluation of a Steerable Magnetic Sheath for Cardiac Ablations
}

\section{Journal Article}

Author(s):

Chautems, Christophe; Lyttle, Sean; Boehler, Quentin (D); Nelson, Bradley J.

Publication date:

2018-07

Permanent link:

https://doi.org/10.3929/ethz-b-000318414

Rights / license:

In Copyright - Non-Commercial Use Permitted

Originally published in:

IEEE Robotics and Automation Letters 3(3), https://doi.org/10.1109/LRA.2018.2809546

Funding acknowledgement:

165564 - Soft Magnetic Robots: Modeling, Design and Control of Magnetically Guided Continuum Manipulators (SNF) 743217 - Soft Micro Robotics (EC) 


\title{
Design and Evaluation of a Steerable Magnetic Sheath for Cardiac Ablations
}

\author{
Christophe Chautems, Sean Lyttle, Quentin Boehler and Bradley J. Nelson
}

\begin{abstract}
Precise catheter control is crucial to the success of radiofrequency cardiac arrhythmia ablations. Remote control using external magnetic fields to directly steer the catheter tip is a promising strategy to accurately and easily perform cardiac catheter steering. Magnetic catheters must be flexible enough to to be deflected by a magnetic field but rigid enough to provide sufficient force and stability during ablation. These conflicting requirements have limited the design options and effectiveness of magnetic catheters. Furthermore, the ablation force achievable by magnetic catheters depends strongly on the catheter's bending angle; at angles greater than 90 degrees it can be very difficult to achieve any significant force during ablation. To overcome these shortcomings, we propose to magnetically control a flexible segment at the distal tip of the introducer sheath, rather than the catheter. Control of the tip orientation is thus decoupled from catheter insertion, enabling more robust and intuitive manipulability of the catheter. Usability testing with the Aeon Phocus, a magnetic navigation system, has shown that the control of this magnetic sheath is indeed more intuitive than the control of a standard magnetic catheter.
\end{abstract}

Index Terms-Surgical Robotics: Steerable Catheters/Needles, Medical Robots and Systems, Flexible Robots

\section{INTRODUCTION}

$\mathbf{R}$ ADIOFREQUENCY (RF) ablation is the treatment of choice for many types of cardiac arrhythmias. A successful ablation treatment replaces life-long antiarrhythmicdrug therapy [1]. The two most common cardiac arrhythmias are atrial flutter (AFL) and atrial fibrillation (AFib) [2]. It has been shown that the pulmonary veins located in the left atrium play a major role in triggering AFib [3]. As a result, electrical isolation of one or more pulmonary veins has become the most common technique for AFib treatment, as depicted by the ablation trajectory in Fig. 1A for the two left pulmonary veins. The most common type of AFL can be treated by a linear lesion from the inferior vena cava (IVC) to the tricuspid ring (see Fig. 1B). The tricuspid ring is the region around the tricuspid valve connecting the right atrium to the right

Manuscript received: September 8, 2017; Revised December 19, 2017; Accepted February 8, 2018.

This paper was recommended for publication by Editor Ken Masamune upon evaluation of the Associate Editor and Reviewers' comments. This work has been partially supported by the Swiss National Science Foundation through grant number 200021_165564, by the Korea Evaluation Institute of Industrial Technology (KEIT) funded by the Ministry of Trade, Industry, and Energy (MOTIE) (no. 10052980), and by the ERC Advanced Grant 743217 Soft Micro Robotics (SOMBOT).

Christophe Chautems, Sean Lyttle, Quentin Boehler and Bradley J. Nelson are with the Multi-Scale Robotics Lab, ETH Zurich, Zurich 8092, Switzerland (e-mail: chautemc@ethz.ch; lyttles@ethz.ch; qboehler@ethz.ch; bnelson@ethz.ch).

Digital Object Identifier (DOI): see top of this page.
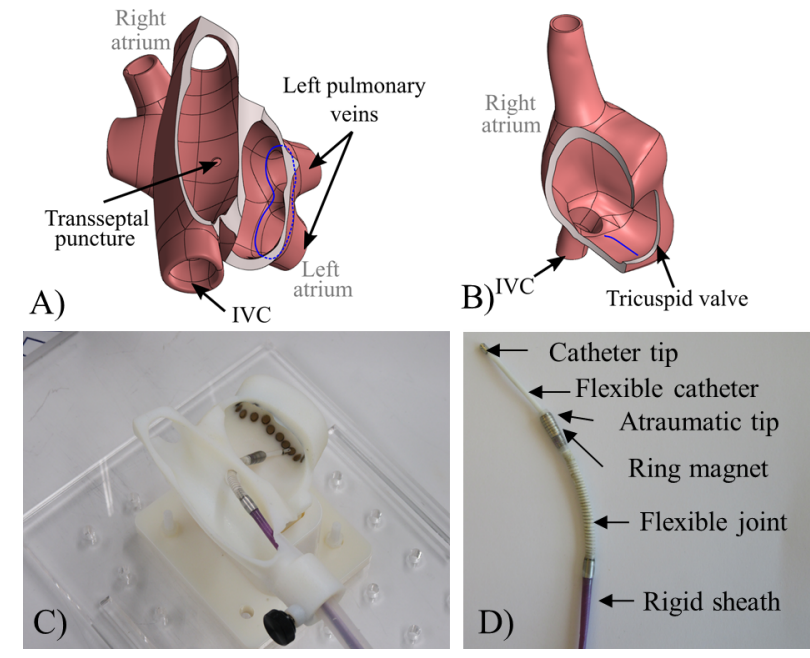

Fig. 1. Overview of AFib and AFL ablations using a magnetic sheath. A) and B) Ablation locations in the left atrium for AFib ablation, and in the right atrium for AFL ablation respectively (ablation trajectories in blue). C) Magnetic sheath inside a 3D printed model of the left atrium with AFib ablation spots. D) Prototype of the magnetic sheath with a custom flexible catheter inserted into the sheath lumen.

ventricle. This linear lesion disrupts the reentrant circuit that initially triggers the arrhythmia [4].

During a standard ablation procedure, a pre-curved introducer sheath is first inserted into the femoral vein all the way up to the right atrium via the IVC. In the case of an AFib ablation, the left atrium is reached from the right atrium via a transseptal puncture performed on the interatrial septum that separates the two atria (see Fig. 1A). The RF ablation catheter is then inserted through the sheath, its tip is steered to reach the ablation target site, and RF energy is delivered through the tip to ablate the tissue. The majority of current commercial catheters are manually controlled by translating and rotating the catheter along its axis via guidewires connected to a proximal handle as depicted in Fig. 2A. A knob on the handle allows bending of the flexible end of the catheter through two antagonistic cables. A large variety of sheaths with different pre-curved shapes are commercially available in order to reach all possible arrhythmia locations. The success rate of the ablation with this standard manual approach has been reported to be between 90 to 95 percent for AFL ablation, and between 50 to 70 percent for AFib ablation [5].

During a complex procedure, it may be required to change the sheath intra-operatively in order to change the distal angle of the sheath. Steerable sheaths have thus been developed to 
avoid such exchanges. A steerable sheath whose tip bending can be controlled may replace the need for catheter tip bending and improve the contact between the ablation tip and the heart tissue, resulting in a lower recurrence of a cardiac arrhythmias as evaluated in [6], [7] with the Agilis steerable sheath (St Jude Medical, St Paul, MN). Two robotic systems have also been developed to control steerable sheaths, the Sensei X2 Robotic System from Hansen Medical [8] and the V-CAS Deflect from Stererotaxis [9]. The use of robotic steerable sheaths has been reported to increase catheter stability, tissue contact, and improve ablation outcome [10].

As an alternative to the classic cable actuation of catheter tips, magnetically actuated catheters are also being used to perform cardiac ablations [11]. This approach is depicted in Fig. 2B. The catheter tip orientation is controlled by generating an external magnetic field $\mathbf{B}$ to apply magnetic torques on magnets disposed along the flexible distal end of the catheter. Several types of magnetic navigation system (MNS) have been developed to generate this field and steer magnetic catheters inside the body. The most widely used system is the Stereotaxis Niobe (Stereotaxis, St. Louis, MO, USA) with more than 150 installations worldwide [12]. Studies have shown the advantage of an MNS for navigation to sites with difficult anatomic locations as well as reduced X-Ray exposure for the physician [13], [14].

Over the past decade, a large number of cardiac arrhythmias have been treated using magnetically steered catheters to perform RF ablations. Magnetic catheters are currently available for clinical use from Biosense Webster (Diamond Bar, CA, USA) and MedFact Engineering GmbH (Lörrach, Germany). Each of these catheters are composed of a series of magnets and flexible segments at the distal tip (see Fig. 2B), and each has limited independence in position and orientation control. In a recent editorial, J. Burkhart pointed out that one of the main limitations of remote magnetic navigation is the limited variety of magnetic catheters [15].

To address the lack of diversity in magnetic tool design, we have previously proposed the concepts of an extremely flexible catheter [16] that can be controlled with a magnetic field and gradient to achieve position and orientation control (5DOF). This approach shows some limitations in term of the magnitude of force achievable with a magnetic gradient. Another alternative is to add variable stiffness sections that can selectively change their stiffness using the phase change of a low melting point alloy [17]. In light of the large number of minimally invasive surgeries with different requirements, there is a need for new magnetic tools that better address the diverse requirements for these procedures. This paper introduces a magnetically steered sheath as an innovative magnetic tool. Much like the manually steerable sheaths, the advantage of the magnetic sheath compared to a magnetic catheter is a decoupling of the motion between tip orientation control and catheter insertion and retraction along the sheath axis, as well as a more direct application of force at large angles. In comparison to the manual sheath, the magnetic sheath has the advantage of applying a magnetic torque directly at the distal end of the sheath and does not require transfer of rotational motion along a long shaft. The expected outcome is

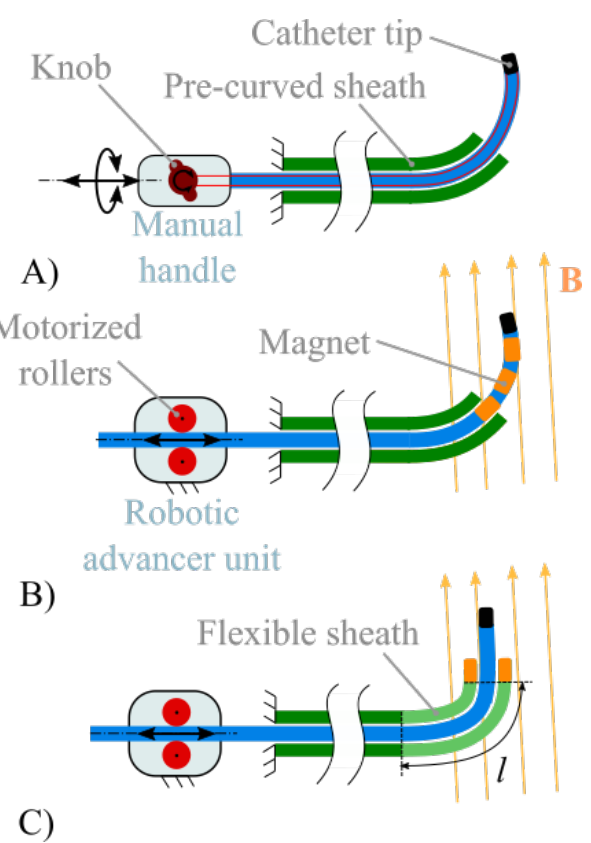

Fig. 2. Approaches for cardiac catheter steering. A) Manual catheter. B) Magnetic catheter. C) Magnetic sheath. Catheter is represented in blue, sheath in green, and magnets in orange.

improvement in the success rate of AFib and AFL ablations, and a decrease in procedure time.

In this paper, the design of the magnetic sheath is first introduced. Its performance is then evaluated and compared with commercial magnetic catheters with an emphasis on its motion control. The use of the magnetic sheath for realistic trajectories performed during AFib and AFL ablations is then demonstrated within a clinical MNS.

\section{MAGNETIC SHEATH}

\section{A. Mechanical Design}

The steerable magnetic sheath is depicted in Fig. 1D. It consists of three sections: a fairly rigid tube spanning the proximal length, an extremely flexible kink-resistant section, and a rigid, magnetically active distal tip section. By magnetically steering the sheath instead of the ablation catheter itself, the ablation catheter's path is constrained, ensuring that all forces applied by the catheter are directly along the long axis of the catheter (see Fig. 2C).

Two magnetic sheath prototypes were constructed. The first was a simple proof-of-concept for preliminary functional testing. The second, depicted in Fig. 1D, is designed to be as close as possible to a functional medical product. Both prototypes have the same outer diameter $(4 \mathrm{~mm})$ and inner diameter $(3 \mathrm{~mm})$. Both sheaths accept catheters of diameter $8 \mathrm{Fr}(2.67 \mathrm{~mm})$ and lower. These diameters are among the most common for cardiac sheaths and catheters. Although the bending length and stiffness of the two prototypes are slightly different, their kinematics are fundamentally the same.

The first prototype's flexible joint was formed by wrapping Teflon tape loosely around a very weak extension spring (25 mm). The proximal section is a short length of a standard 
St. Jude braided introducer sheath. The distal section comprises eight ring magnets pressed onto a plastic tube.

In the second prototype, the first rigid section was extended to correspond to the length of the access path from the groin to the right atrium. The distance from the proximal hemostatic valve adapter to the beginning of the flexible joint section is $650 \mathrm{~mm}$. This section is made from off-the-shelf braided tubing that is commonly used in cardiac sheaths. The flexible joint section $(35 \mathrm{~mm})$ consists of an extremely flexible stainless steel extension spring coated in a thin layer of expanded PTFE (ePTFE). The ePTFE coating encloses the spring without adding much stiffness. There are other possible embodiments of the flexible joint, including a corrugated section like that in a drinking straw, or concentric rings contained in an elastic polymer, but the spring wrapped with ePTFE was found to be the simplest and most robust solution. The distal tip of the sheath $(13 \mathrm{~mm})$ consists of a small section of rigid braided tube, eight permanent ring magnets, and an atraumatic tip. The three sections of the second prototype are crimped together with stainless steel collars.

In order to optimize the performance of the magnetic sheath, an extremely flexible catheter was also constructed. This catheter allows the magnetic sheath to bend freely. Axial stability is provided by the magnetic sheath and not by the catheter itself as in the standard case.

\section{B. Interoperability with Commercial Advancer Unit and Catheters}

The translation of a magnetic catheter inside a traditional introducer sheath is performed by a robotic advancer unit [18] which consists of two motorized rollers (see Fig. 2). The magnetic sheath and catheter have been designed to be interoperable with the advancer unit commercialized by Aeon Scientific. It can be operated in the same way as for a fixed curvature standard sheath with the advancer unit inserting and retracting the flexible catheter inside the magnetic sheath lumen.

The magnetic sheath has an inner diameter identical to common insertion sheaths and allows the insertion of a traditional catheter. During a procedure it is common to use multiple catheters, for example a mapping catheter to capture the heart chamber geometry. A mapping catheter can be inserted directly inside the magnetic sheath. The possibility to use a manual catheter is still available as it can also be inserted into the magnetic sheath. The magnetic sheath will slightly influence the mobility of the manual catheter and it will not behave in exactly the same way as with a straight sheath. The flexible section will bend with the catheter and the rigid magnet will keep a short section of the catheter straight, but this will not significantly alter the behavior of the manual catheter.

\section{Magnetic Sheath Performance}

\section{A. Control of a Magnetic Tool}

Each magnetic dipole $\mathbf{m}$ inside a magnetic field $\mathbf{B}$ is subjected to a magnetic torque $\mathbf{T}$ and a magnetic force $\mathbf{F}$.

$$
\mathbf{F}=(\mathbf{m} \cdot \nabla) \mathbf{B}
$$

$$
\mathbf{T}=\mathbf{m} \times \mathbf{B}
$$

The direction of the magnetic field is conveniently expressed in spherical coordinates with the azimuthal direction aligned with the base orientation. The magnetic field at one location is then fully defined by an inclination angle $\theta$, an azimuthal angle $\phi$, and the magnetic field magnitude as depicted in Fig. 3.

A large MNS has a workspace center located approximately $300 \mathrm{~mm}$ from the electromagnet tips. Because the magnets on the catheter are at such close proximity $(\approx 10 \mathrm{~mm})$ to each other, and so far from the electromagnets, they are exposed to a similar magnetic field. For this reason, we assume a uniform and constant magnetic field in our working volume. Thus for modeling purposes, we only consider the magnetic torque on the catheter. The magnetic force is neglected as it is created by the magnetic field gradients that are assumed to be small in a large MNS.

To verify this assumption, experimental data were collected. A magnetic catheter was positioned in an MNS with the sheath's proximal side constrained along the horizontal main axis of the system. Tip positions were recorded by fixing two color markers on the tip and using two cameras to track the markers. The marker centroids were extracted from the camera image using standard image processing tools. The tip position is conveniently expressed in cylindrical coordinates with the longitudinal axis collinear to the sheath base axis. The catheter configuration is thus described by the longitudinal coordinate $Z$, the radial coordinate $R$ and the azimuthal angle $\phi$ as depicted in Fig. 3. The magnetic field inclination angle and the azimuthal angle were increased by steps of $30^{\circ}$ to cover the full azimuthal range $0^{\circ}$ to $360^{\circ}$ and inclination between $30^{\circ}$ to $150^{\circ}$ (magnetic field magnitude $\approx 120 \mathrm{mT}$ ). The catheter length was increased by $20 \mathrm{~mm}$ insertion steps within the sheath.

Measurements with identical catheter lengths and magnetic field inclination angles form a cluster independent of magnetic field azimuthal angles, as depicted in Fig. 3. The mean distance from the cluster median is $2.7 \mathrm{~mm}$ and the standard deviation is $3.0 \mathrm{~mm}$. These clusters validate our assumptions of rotational symmetry, meaning that the behavior of a magnetic tool can be assessed within a single azimuthal plane. The influence of gravity and magnetic force were too insignificant to measure, validating our assumption that they can be neglected.

Using the model based on these two assumptions proposed by I. Tunay [19], for a thin body deflected by a magnetic field, the flexible segment constant curvature $\kappa$ is given by

$$
\kappa=\frac{T_{m}}{E I}
$$

where $T_{m}$ is the bending moment, $E$ is the modulus of elasticity in tension, and $I$ is the area moment of inertia. In the plane of deflection, the distal segment orientation relative to the proximal segment orientation is represented by $\theta_{t i p}$ and the magnetic field inclination angle is represented by $\theta$. The 


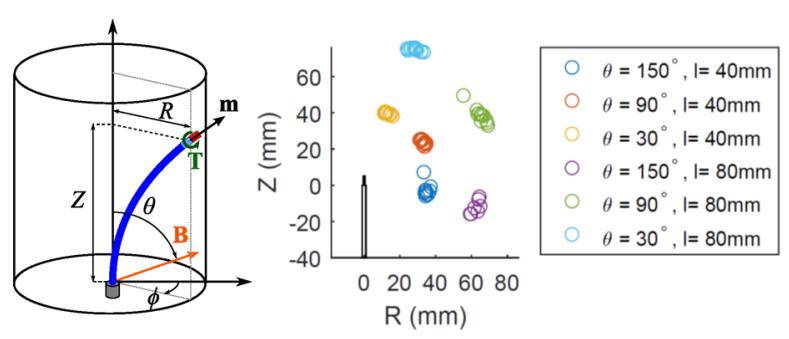

Fig. 3. Parametrization of the magnetic tool inside a magnetic field. Magnetic field in orange, magnetic tool in blue and azimuthal plane in gray dotted line On the right, tip positions of a commercial Trignum magnetic catheter for different catheter insertion lengths and magnetic field directions in cylindrical coordinates.

conversion between tip and curvature is given by $\theta_{t i p}=\kappa l$. With the magnetic field equation (1) inserted into (3), we get:

$$
\theta_{\text {tip }}=\frac{l B M}{E I} \sin \left(\theta-\theta_{t i p}\right)
$$

The resulting tip orientation and position can be computed using a numerical solver. This can be extended to multiple magnetic segments by having one equation per magnet and summing the magnetic torque acting on each flexible segment. The set of equations can then be solved numerically.

\section{B. Kinematic Comparison with Magnetic Catheters}

Magnetic catheters, as depicted in Fig. 2B, suffer from several important kinematic disadvantages. First, the distance from the tip of the sheath to the magnetic segments changes depending on the catheter's insertion length, i.e $l$ is not constant in equation (4). Therefore, in a constant magnetic field, the curvature of the catheter will change as it is inserted or retracted, making control somewhat complex. Second, the stiffness of the magnetic catheter changes as it is inserted and retracted, i.e. $E$ in equations (3) and (4) is also a function of $l$. This is due to the difference in stiffness between the flexible sections and the rigid magnetic sections. Furthermore, because commercial magnetic catheters have multiple distal magnets, the amount of magnetic volume $M$ also changes with insertion length.

The magnetic sheath addresses these limitations by magnetically controlling the sheath instead of the catheter, thus decoupling curvature and insertion. Because the length of the sheath's flexible distal section does not change ( $l$ is constant), the curvature of this section does not depend on the insertion length of the catheter. Also, the magnet location and flexibility of the sheath are constant and wholly decoupled from the insertion length of the catheter. Furthermore, because the magnetic sheath creates a path the catheter must follow, the direction of the force applied by the catheter will always be coaxial with the tip of the sheath, which can be advantageous for contact force and stability at large bending angles.

Figure 4 shows the results of kinematic simulations in MATLAB for the magnetic catheter and magnetic sheath. The nonlinear behavior of the magnetic catheter is clearly seen, in contrast with the linear behavior of the magnetic sheath.

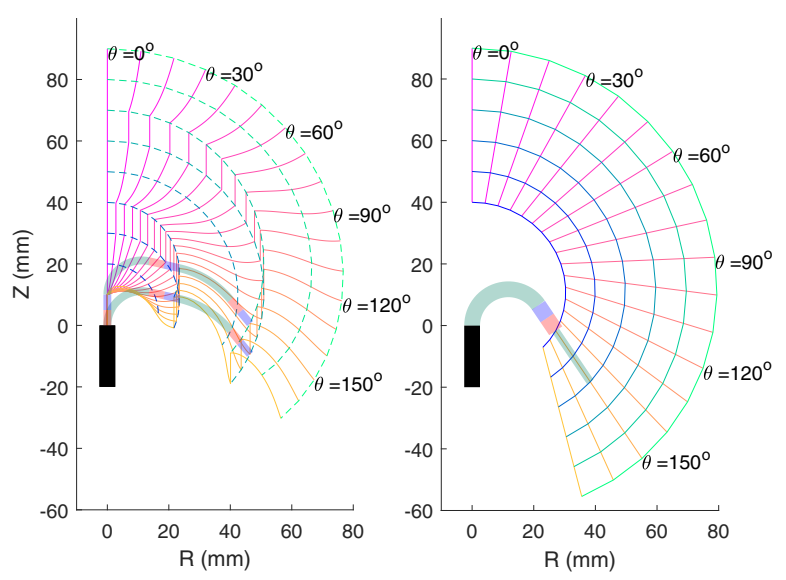

Fig. 4. Kinematic comparison of a three-magnet magnetic catheter (left plot) and the magnetic sheath (right plot). Pink-orange lines represent tip positions for a given magnetic field inclination angles and green-blue lines represent tip positions for fixed catheter insertion lengths. For the magnetic catheter the line with constant magnetic field but varying catheter length shows a sudden change of direction. This is not the case for the magnetic sheath. The flexible section of the magnetic sheath is $30 \mathrm{~mm}$ long and the magnet $10 \mathrm{~mm}$. The three-magnet catheter has a flexible section length of $20 \mathrm{~mm}$ and a magnet length of $10 \mathrm{~mm}$.

\section{Experimental Results}

In order to confirm the simulation results of the kinematic model, experimental data were collected on two commercial catheters and the magnetic sheath. The commercial products considered for this comparison are a Trignum magnetic catheter (Biotronik) and a MagnoFlush magnetic catheter (MedFact Engineering GmbH), both composed of three rigid magnets placed along the flexible distal part of the catheter. The magnetic sheath used for these experiments was the second prototype with a $35 \mathrm{~mm}$ flexible section. With a constant magnetic field direction and magnitude, the catheter was incrementally inserted through the sheath in steps of $2 \mathrm{~mm}$, and the position of the tip was measured with a sub-mm visual tracking system. A inclination angle of $150^{\circ}$ was selected for these tests.

The results are depicted in Fig. 5. Nonlinear behavior was observed for both commercial catheters. Five different configurations of the MagnoFlush catheter along this curve are represented (see top of Fig. 5). The nonlinearity observed between configurations 1) and 2) corresponds to the situation where the second flexible segment of the catheter (in white) comes out of the straight sheath. In this case a $2 \mathrm{~mm}$ insertion can lead to a $15 \mathrm{~mm}$ tip displacement. When a rigid magnet comes out of the sheath, the insertion curve exhibits a change of tip motion direction. These effects clearly appear for both commercial catheters and are successfully avoided with the magnetic sheath as magnetic steering is performed on the sheath itself rather than on the catheter (see Fig. 2B and C). With this approach, the insertion of the catheter within the sheath does not affect its distal orientation meaning that the insertion and orientation motion are decoupled.

\section{User Control for Cardiac Ablations}

During an ablation procedure, the electrophysiologist observes the catheter via X-ray images or using a commercially 

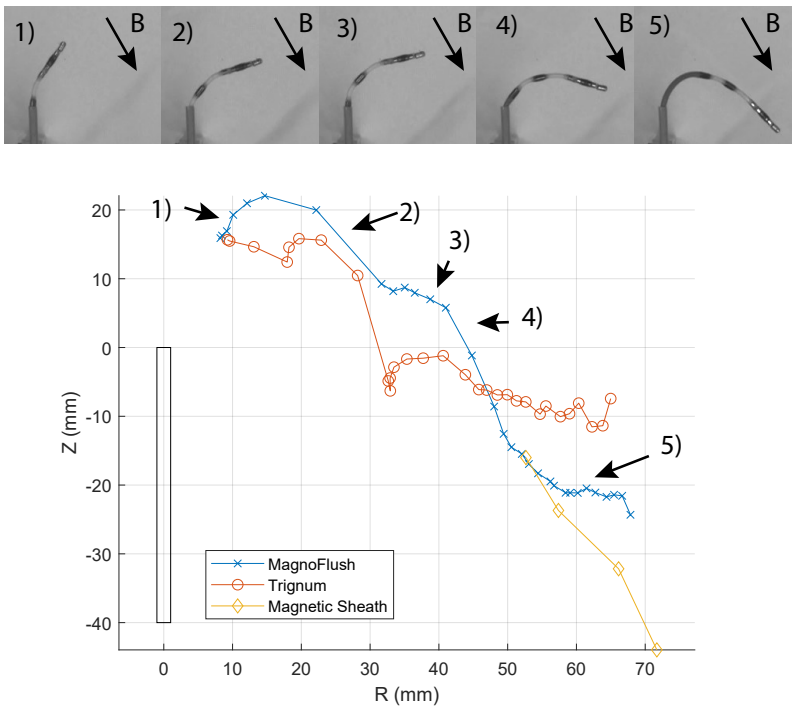

Fig. 5. Catheter tip position for a magnetic field inclination $\theta=150^{\circ}$ for MagnoFlush catheter, Trignum catheter, and first magnetic sheath prototype. The measurements are every $2 \mathrm{~mm}$ for the MagnoFlush and Trignum catheter and every $10 \mathrm{~mm}$ for the magnetic sheath. Due to the color marker on the tip, no data is available for a catheter length below $20 \mathrm{~mm}$. Five pictures illustrate different stages of the MagnoFlush catheter insertion curve.

available EP localization mapping system (e.g. Carto3 or Ensite Navx). In newer mapping systems, the catheter tip position is displayed on a 3D heart map, and ECG signals are overlaid on top of the heart map. These mapping systems are used with manual or magnetic catheters. The electrophysiologist can then control the catheter motion using this visual information.

The magnetic sheath prototypes were also tested inside a clinically available MNS (Aeon Phocus, Aeon Scientific AG). A 3D joystick allows the user to rotate the magnetic field. Two buttons on the joystick control the robotic advancer unit for insertion and retraction of the ablation catheter inside the sheath. The magnetic field direction and the insertion length are displayed within a dedicated graphical user interface.

Two 3D-printed models were used to evaluate the ability of the magnetic sheath to reach target sites for AFL and AFib ablation. Of course the 3D-models do not fully simulate the conditions of a real cardiac environment, such as blood flow and tissue deformation. However, their geometries are realistic enough to evaluate the ability of the magnetic tools to follow a realistic ablation trajectory. Snapshots of the procedures are depicted for both cases in Fig. 6. The models are open on one side to allow the user to observe the magnetic sheath motion directly.

In the case of AFL ablation, the ablation targets are located around the tricuspid ring which is difficult to reach when coming from the IVC, as it requires bending the tool close to $180^{\circ}$ (see Fig. 1B). It is extremely challenging to reach the ablation spots with a magnetic catheter in the $3 \mathrm{D}$ right atrium model. Due to the decoupling of the catheter insertion and orientation, the trajectory is much easier with the magnetic sheath. The sheath is first steered to point the tip toward the ablation site near the IVC, then the catheter can be inserted through the sheath with a stable fixed tip orientation. This way,
TABLE I

TIME IN MINUTES AND SECONDS TO REACH THE 19 AFIB ABLATION POINTS WITH EXPERIMENT REPEATED SIX TIMES.

\begin{tabular}{|c|c|c|c|}
\hline & Mean Time & Min & Max \\
Biosense catheter & 6'55' & 5'27’ & $8^{\prime} 30^{\prime \prime}$ \\
Magnetic sheath & 3'14' & 2'31' & 3'51' \\
\hline
\end{tabular}

the catheter maintains steady contact with the heart model wall, and the contact force is directly perpendicular to the target site. The first sheath prototype with a $25 \mathrm{~mm}$ flexible section was used for the AFL experiments, because it has a slightly smaller bending radius.

In AFib ablation, the pulmonary veins located in the left atrium are usually the targets for ablation. Access to the left atrium requires a transeptal puncture perforating the wall between the right and the left atrium. AFib ablation tests were performed with our second prototype inside the CardioMag MNS (Aeon Phocus non-medical prototype). As in the AFL model, navigation of the magnetic sheath appeared more intuitive than that of the magnetic catheter because of the decoupling of the catheter insertion and sheath orientation. This was quantitatively verified by measuring the mean time to reach 19 ablations points, which was two times faster compared with the Biosense catheter (Table I). Based on visual observation, the position accuracy and path-following ability of the magnetic sheath was qualitatively similar to that of a magnetic catheter, although a precise quantification would require accurate localization feedback integrated into the catheter.

The users suggested the following improvements of the control functionality:

- Ability to go back to a previous position (position history)

- Linear interpolation between start and end of an ablation path

These features were implemented into our research framework. The user was able to record the trajectories and move back to a previous position. In a majority of cases, the user only wanted to move back to an orientation and manually control the insertion, so the ability to reach a previous orientation without changing catheter length is a useful feature. Another advantage of controlling only the magnetic field orientation is that we avoid any risk of perforation during motion. Besides the ablation step, the insertion step is the one that can most often result in a perforation in adverse situations. However, even this risk is minimal as the soft tip of a magnetic catheter should not be able to apply sufficient force to perforate healthy tissue.

Linear interpolation was also implemented. The user was able to select an initial and final magnetic sheath orientation. The user could then move between the two orientations by small increments. This functionality allows for a more intuitive motion of the catheter tip along the linear trajectory necessary for an AFL ablation.

\section{CONCLUSion}

We have presented the design and evaluation of a new magnetic sheath for cardiac ablations. Due to its kinematics, its 


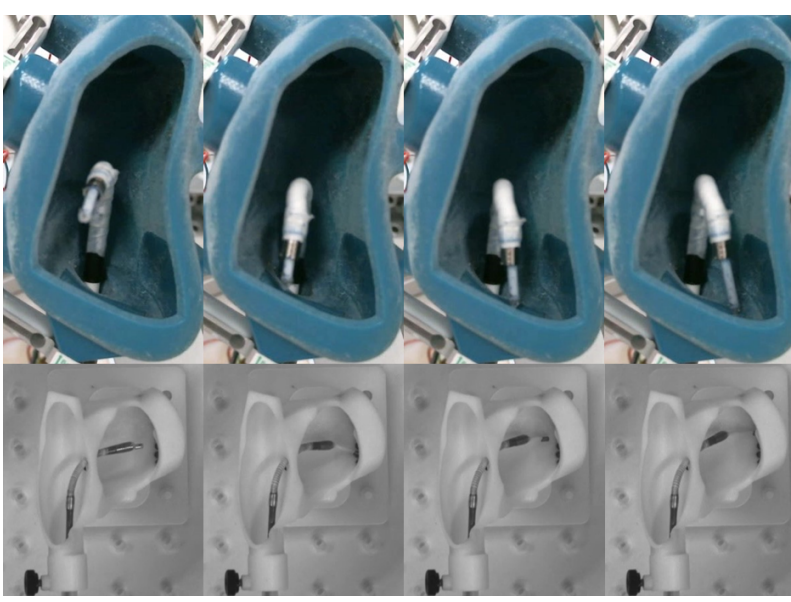

Fig. 6. Magnetic sheath manipulation in 3D printed model for testing AFL ablation (top) and AFib ablation (bottom).

motion control is greatly simplified compared to commercial magnetic catheters. This is primarily because the orientation of the catheter tip, controlled by the magnetic field direction, is decoupled from its insertion motion within the sheath.

Preliminary tests on heart models were carried out to target common ablation locations for AFL and AFib procedures. Control of the magnetic sheath within a medical MNS was shown to be simpler and faster compared to a magnetic catheter. This promises more intuitive control for the electrophysiologist, and thus a reduction in procedure time.

\section{REFERENCES}

[1] F. Morady, "Radio-Frequency Ablation as Treatment for Cardiac Arrhythmias," New England Journal of Medicine, vol. 340, no. 7, pp. 534-544, 21999.

[2] R. K. Mareedu, I. B. Abdalrahman, K. C. Dharmashankar, J. F. Granada, P.-H. Chyou, P. P. Sharma, P. N. Smith, J. J. Hayes, R. T. Greenlee, and H. Vidaillet, "Atrial Flutter Versus Atrial Fibrillation in a General Population: Differences in Comorbidities Associated With Their Respective Onset," Clinical Medicine \& Research, vol. 8, no. 1, pp. 1-6, 32010.

[3] S. Mahida, F. Sacher, N. Derval, B. Berte, S. Yamashita, D. Hooks, A. Denis, S. Amraoui, M. Hocini, M. Haissaguerre, and P. Jais, "Arrhythmia Mechanisms Science Linking Pulmonary Veins and Atrial Fibrillation," Arrhythmia \& electrophysiology review, vol. 4, no. 1, pp. 40-43, 2015.

[4] K. Lee, Y. Yang, and M. Scheinman, "Atrial flutter: A review of its history, mechanisms, clinical features, and current therapy," Current Problems in Cardiology, vol. 30, no. 3, pp. 121-167, 32005.

[5] J. P. Joseph and K. Rajappan, "Radiofrequency ablation of cardiac arrhythmias: past, present and future," QJM, vol. 105 , no. 4, pp. 303314, 42012.

[6] C. Piorkowski, C. Eitel, S. Rolf, K. Bode, P. Sommer, T. Gaspar, S. Kircher, U. Wetzel, A. S. Parwani, L.-H. Boldt, M. Mende, A. Bollmann, D. Husser, N. Dagres, M. Esato, A. Arya, W. Haverkamp, and G. Hindricks, "Steerable Versus Nonsteerable Sheath Technology in Atrial Fibrillation AblationClinical Perspective," Circulation: Arrhythmia and Electrophysiology, vol. 4, no. 2, 2011.

[7] S. Matsuo, T. Yamane, M. Tokuda, T. Date, M. Hioki, R. Narui, K. Ito, S. Yamashita, Y. Hama, T. Nakane, K. Inada, K. Shibayama, S. Miyanaga, H. Yoshida, H. Miyazaki, K. Abe, K.-i. Sugimoto, I. Taniguchi, and M. Yoshimura, "Prospective randomized comparison of a steerable versus a non-steerable sheath for typical atrial flutter ablation," Europace, vol. 12, no. 3, pp. 402-409, 32010.

[8] P. Kanagaratnam, M. Koa-Wing, D. T. Wallace, A. S. Goldenberg, N. S Peters, and D. W. Davies, "Experience of robotic catheter ablation in humans using a novel remotely steerable catheter sheath," Journal of Interventional Cardiac Electrophysiology, vol. 21, no. 1, pp. 19-26, 1 2008.
[9] A. Errahmouni, D. G. Latcu, S.-S. Bun, N. Rijo, C. Dugourd, and N. Saoudi, "Remotely controlled steerable sheath improves result and procedural parameters of atrial fibrillation ablation with magnetic navigation," Europace, vol. 17, no. 7, pp. 1045-1050, 72015.

[10] L. C. Malcolme-Lawes, P. B. Lim, M. Koa-Wing, Z. I. Whinnett, S. Jamil-Copley, S. Hayat, D. P. Francis, P. Kojodjojo, D. W. Davies, N. S. Peters, and P. Kanagaratnam, "Robotic assistance and general anaesthesia improve catheter stability and increase signal attenuation during atrial fibrillation ablation," Europace, vol. 15, no. 1, pp. 41-47, 12013.

[11] A. Ali, D. Plettenburg, and P. Breedveld, "Steerable Catheters in Cardiology: Classifying Steerability and Assessing Future Challenges," IEEE Transactions on Biomedical Engineering, pp. 1-1, 2016.

[12] C. Pappone, G. Vicedomini, F. Manguso, F. Gugliotta, P. Mazzone, S. Gulletta, N. Sora, S. Sala, A. Marzi, G. Augello, L. Livolsi, A. Santagostino, and V. Santinelli, "Robotic magnetic navigation for atrial fibrillation ablation," Journal of the American College of Cardiology, vol. 47, no. 7, pp. 1390-1400, 2006.

[13] M. Kawamura, M. M. Scheinman, Z. H. Tseng, B. K. Lee, G. M. Marcus, and N. Badhwar, "Comparison of remote magnetic navigation ablation and manual ablation of idiopathic ventricular arrhythmia after failed manual ablation," Journal of Interventional Cardiac Electrophysiology, pp. 1-8, 62016.

[14] A. Aryana, A. d'Avila, E. K. Heist, T. Mela, J. P. Singh, J. N. Ruskin, and V. Y. Reddy, "Remote magnetic navigation to guide endocardial and epicardial catheter mapping of scar-related ventricular tachycardia." Circulation, vol. 115, no. 10, pp. 1191-200, 32007.

[15] J. D. Burkhardt, "Remote magnetic navigation for ventricular ablation: did the machine win this round?" Journal of Interventional Cardiac Electrophysiology, vol. 48, no. 1, pp. 5-7, 12017.

[16] C. Chautems and B. J. Nelson, "The tethered magnet: Force and 5DOF pose control for cardiac ablation," in 2017 IEEE International Conference on Robotics and Automation (ICRA). IEEE, 5 2017, pp. $4837-4842$.

[17] C. Chautems, A. Tonazzini, D. Floreano, and B. J. Nelson, "A Variable Stiffness Catheter Controlled with an External Magnetic Field," 2017 IEEE/RSJ International Conference on Intelligent Robots and Systems, 2017.

[18] S. Ernst, "Remote Catheter Navigation Systems," in Catheter Ablation of Cardiac Arrhythmias, 2011, ch. 9, pp. 137-145.

[19] I. Tunay, "Position control of catheters using magnetic fields," in Proceedings of the IEEE International Conference on Mechatronics, 2004. ICM '04. IEEE, pp. 392-397. 